\title{
Immune-Based Modulation of Adult Hippocampal Neurogenesis, Link to Systemic Th1/Th2 Balance
}

Fangfang Qi, Yucen Xia, Zejie Zuo, Yingying Wu, Junhua Yang, Xiao Wang and Zhibin Yao*

Department of Anatomy and Neurobiology, Zhongshan School of Medicine, Sun Yat-sen University, \#74, Zhongshan No. 2 Road, Guangzhou 510080, PR China

*Corresponding author: Zhibin Yao, Department of Anatomy and Neurobiology, Zhongshan School of Medicine, Sun Yat-sen University, \#74, Zhongshan No. 2 Road, Guangzhou 510080, PR China, Tel: + 8620 87332638; Fax: + 8620 87330709; E-mail: yao.zb@163.com

Received date: 23 December 2014; Accepted date: 11 February 2015; Published date: 16 February 2015

Copyright: @ 2015 Qi F, et al. This is an open-access article distributed under the terms of the Creative Commons Attribution License, which permits unrestricted use, distribution, and reproduction in any medium, provided the original author and source are credited.

\begin{abstract}
The interaction between adaptive immune system and the Central Nervous System (CNS) has been extensively studied for decades. A series of researches have indicated that systemic $T$ cells, particularly $C D 4^{+} T$ cells are involved in supporting fundamental processes of brain functional integrity, such as in the maintenance of brain plasticity including spatial learning and memory, and neurogenesis. Moreover, recent data from our laboratory indicate alteration of systemic T helper cell type 1 (Th1)/Th2 balance is tightly linked to hippocampal neurogenesis and working memory. In this review, we summarize current knowledge of the systemic Th1/Th2 balance, describe how this skewing appears to operate in hippocampal neurogenesis, and reinforce the theory of interaction mechanism of these two vital systems.
\end{abstract}

Keywords: Th bias; Dentate gyrus; Immune system; Correlation; Cytokine; Neurogenesis

\section{Introduction}

Brain has not been considered as a classic immunologically privileged organ recent years [1,2], in which, peripheral immune cells could not through the blood brain barrier into the CNS under normal physiological state [3]. Current researches in neuroimmune interactions focus on immune cytokines- or cells-based regulation of cognitive function and neural plasticity rather than traditional brain damage [4]. Hippocampal neurogenesis continues in the Dentate Gyrus (DG) of the hippocampus throughout adult life. Adaptive peripheral immune activation regulates proliferation of neural precursor cells in adult hippocampus [5-7]. Recent researches also provide excellent evidence that stress-induced hippocampal neurogenesis alteration in DG is accompanied by Th1/Th2 balance changing in the periphery $[8,9]$. Together with our recent studies $[10,11]$, we have proposed a concept that underlying mechanism of immune system affecting the CNS is not just regulated by a certain cytokine but rather regulated by many cytokines [12], which are correlated with systemic Th1/Th2 cytokine balance, for modulation of elaborated brain functions. Importantly, other reports indeed shed light on the correlation between systemic Th1/Th2 balance and adult hippocampal neurogenesis $[8,13]$; although recent data indicate that the dichotomous categorization of helper T lymphocyte into Th1 and Th2 has become gradually blurred. We purpose the hypothesis that the alterations in adult hippocampal neurogenesis could be related to a differential regulation of systemic Th1/Th2 cytokine balance. In this article, we focused on some of the most recent results on systemic Th1/Th2 bias within a normal range on adult hippocampal neurogenesis and review results obtained in our laboratory. We summarize first, the effects of systemic Th1 bias on adult hippocampus; second the effects of Th2 bias on adult hippocampus; thirdly, how and where Th1/Th2 balance influences adult hippocampal neurogenesis. We end with a supposition of the effects of excessive helper $\mathrm{T}$ cell bias on hippocampal neurogenesis under pathological conditions.

\section{Th1 bias and adult hippocampal neurogenesis}

Under normal or physiological condition, it has been demonstrated that systemic Th1 bias is correlated with adult hippocampal neurogenesis. A model of modest exercise-training, which is widely recognized to increase adult hippocampal neurogenesis, causes Th1 bias via up-regulating interferon gamma (IFN- $\gamma$ ) levels in the periphery [11]. We recently showed that influenza A (H1N1) vaccination during early pregnancy transiently promote adult hippocampal neurogenesis, potentially via the restoration of the balance of Th1/Th2 [10]. Pregnant mice display a decrease in hippocampal neurogenesis followed by a down-regulation in systemic Th1/Th2 balance [14,15]. However, this neuronal deficit can be reversed by influenza vaccination, which is known to induce Th1 bias during early pregnancy [10]. Importantly, there exists a positive correlation between the interferon (IFN)- $\gamma$ to interleukin (IL)-4 ratio and hippocampal Brain-derived neurotrophic factor (BDNF) levels, further supporting our viewpoint [10]. Furthermore, one mouse model of rheumatoid arthritis used by Wolf, S.A. et al. characterized by a Th1-dependent local inflammation of the knee joints increases proliferation of neural precursor cells in the adult hippocampus [5], as can be seen in Table 1. Interestingly, the intensity of observed change in Th1 bias might be antigen-specific. This is exemplified in a study Teixeira L et al. who observed a strong Th1-type immune response induced by the protozoan parasite [16], which can impair adult neurogenesis reflected by a decline in the neural stem cell pool at peak parasitemia [17-23]. This question was, thus, not within the scope of the present article for its pathological condition. Together, these findings confirm that peripheral immune response such as Th1 bias indeed correlates with adult hippocampal progenitor cells niche, whereby adjusting to cognitive function. However, the range of Th1 bias remains to be explored in further research [24-35]. 
Page 2 of 4

\begin{tabular}{|c|c|c|c|}
\hline Th1/Th2 balance & Objective & Results & References \\
\hline Th1 bias & Study mechanism of stress with cognitive deficit & $\begin{array}{l}\text { Th1-type cytokine correlates to a better } \\
\text { performance }\end{array}$ & Palumboa et al. [9] \\
\hline Th1 bias & $\begin{array}{l}\text { Study association of Glatiramer of adult } \\
\text { neurogenesis }\end{array}$ & $\begin{array}{l}\text { Glatiramer induce Th1 bias } \\
\text { following increasing neurogenesis }\end{array}$ & Palumboa et al., He et al. $[9,13]$ \\
\hline Th1 bias & Study association of exercise of neurogenesis & Moderate exercise produces Th1-like cytokine & Baum et al. [11] \\
\hline Th1 bias & $\begin{array}{l}\text { Study association of } \mathrm{A}(\mathrm{H} 1 \mathrm{~N} 1) \text { Vaccination of } \\
\text { neurogenesis }\end{array}$ & $\begin{array}{l}\mathrm{A}(\mathrm{H} 1 \mathrm{~N} 1) \text { Vaccination causes Th1-like immune } \\
\text { response }\end{array}$ & Xia et al. [10] \\
\hline Th1 bias & $\begin{array}{l}\text { Adaptive immune response increases } \\
\text { hippocampal proliferation }\end{array}$ & $\begin{array}{l}\text { A rheumatoid arthritis model, } \\
\text { Th1-dependent local inflammation }\end{array}$ & Wolf et al. [5] \\
\hline Th2 bias & $\begin{array}{l}\text { Study the effect of zinc on Th1/Th2 ratio in } \\
\text { elderly individuals }\end{array}$ & Decrease of Th1/Th2 ratio with aging & Kahmann et al. [24] \\
\hline Th2 bias & Altered cytokine production in the elderly & Decrease of Th1/Th2 ratio with aging & Rink et al. [22] \\
\hline Th2 bias & $\begin{array}{l}\text { Shift toward destructive Th2 inflammation in } \\
\text { brain }\end{array}$ & $\begin{array}{l}\mathrm{T} \text { helper type } 2 \text { (Th2) in choroid plexus } \\
\text { inflammation with aging }\end{array}$ & Baruch et al. [36] \\
\hline Th2 bias & $\begin{array}{l}\text { Th2 bias at the maternal/fetal interface in } \\
\text { pregnancy }\end{array}$ & $\begin{array}{l}\text { Decrease of neurogenesis in pregnancy related } \\
\text { in Th2 bias }\end{array}$ & $\begin{array}{l}\text { Piao et al. [15] } \\
\text { Rolls et al. [14] }\end{array}$ \\
\hline
\end{tabular}

Table 1: Association of adult hippocampal neurogenesis or cognitive function with Th1/Th2 balance.

\section{Th2 bias and adult hippocampal neurogenesis}

The previously discussed findings clearly show that reestablishing the systemic Th1/Th2 cytokine balance could restore adult hippocampal and neurocognition $[8,13]$. As we expected, Peripheral Blood Mononuclear Cell (PBMC) activated ex vivo with external antigen exhibited an age-related decline in Th1 cell response, shown by the decline in the frequency of IFN- $\gamma$-secreting memory $\mathrm{T}$ cells using Enzyme-Linked Immunospot Assay (ELISPOT) or flow cytometry. The reduced frequency of IFN- $\gamma$-secreting $\mathrm{T}$ cells is accompanied by an increased Th2 response in elderly subjects [18]. So the cognitive impairment observed in aged subjected might be partially explained by increasing destructive Th2 inflammation in the Choroid Plexus (CP) accompany by inducing expression of CCL11 [19], a chemokine elevated in the Cerebrospinal Fluid (CSF) and plasma in the aged human and mice [20]. It is important to note that the Th2 inflammation was observed in normal aging. So it is within the scope of physiological conditions. In line with this, accumulating evidence supports the relationship of decrease in Th1/Th2 cytokine balance and aging-related cognitive decline [21,22]. Interestingly, the prominent $\mathrm{Th} 2$ bias can be reversed by zinc supplementation in aging individuals $[23,24]$, as can be seen in Table 1 . However, whether agingrelated cognitive impairment can be restored after zinc supplementation remains elusive. It requires further studies to elaborate this question.

Besides aging-related Th2 bias, studies indicated that decreased hippocampal neurogenesis may link to Th2 bias at the maternal/fetal interface during pregnancy $[14,15]$. Although IL-4-producing T cells were recently identified as anti-inflammatory cytokine and shown to support neural regeneration under pathological conditions [25,26], high levels of IL-4 induced the choroid plexus to produce CCL11 [19], which is associated with cognitive decline. Notably, the typical cytokine IL-4 is not completely equivalent to the Th2-derived cytokines. Notwithstanding, these data indeed increase the probability that Th2-derived cytokines correlate with decreased hippocampal neurogenesis and cognitive impairment.

\section{How systemic Th1/Th2 cytokine balance modulates adult hippocampal neurogenesis}

Systemic Th1/Th2 cytokine balance is known for their role in many immune responses $[27,28]$. It has recently been implicated in the regulation of adult hippocampal neurogenesis [29-31]. However, the mechanism by which Th1/Th2 influences neuronal proliferation remains unknown. Here we present that the possible cytokines modulate adult hippocampal neurogenesis involved in Th1/Th2 cytokine balance. Therefore, we focus on the role of IFN- $\gamma$ and IL-4, which serve as the classical representative of Th1 cytokines and Th2 cytokines, respectively. For one thing, recent data reveals that the proinflammatory cytokine, IFN- $\gamma$ but not TNF-a, enhances neuronal differentiation, rapidly increasing $\beta$ III-tubulin positive cell numbers in certain vitro culture [32]. Moreover, R. Baron et al. have also demonstrated that IFN- $\gamma$ enhances neurogenesis in the DG and improves the spatial learning abilities both in adult and aged animal models. IFN- $\gamma$ Tg mice performing better spatial cognitive function than naïve mice further confirms the role of IFN- $\gamma$ implicated in the fate of neural precursor cells (NPCs) in the hippocampus [33]. It is important to note that the level of IFN- $\gamma$ is relative low or limited amounts at above experiments. In contrast, high levels of IFN- $\gamma$ inhibits adult hippocampal neurogenesis via the activation of other pro-inflammatory cytokines such as TNF-a, IL-1 $\beta$, IL-6. These results imply that low-level inflammatory cytokines aid neurogenesis but high- level ones impair neurogenesis, reminiscent of cytological changes following homeostatic neurogenic niche [12]. For another thing, the role of IL-4 on neurogenesis and cognitive function is well documented, but the explanations for it are very controversial. Consistent with our results, Nunan $\mathrm{R}$ et al. have well demonstrated that VIP, a neuropeptide released by the interneurons in DG, enhances 
the pro-neurogenic effect of microglia via IL-4 release [34]. However, a dominant shift in favor of Th2 bias identified by the IFN- $\gamma$ : IL-4 ratio indeed regulates negatively hippocampal neurogenesis independent of IL-4 level $[8,10]$. The underlying mechanism by which the Th2 bias regulates hippocampal neurogenesis is incompletely understood. A recent study from our laboratory reveals that increased Th1/Th2 balance transiently promotes neurocognition, probably via the alterations of cytokines expression in peripheral and neurochemicals expression in the hippocampus (unpublished data).

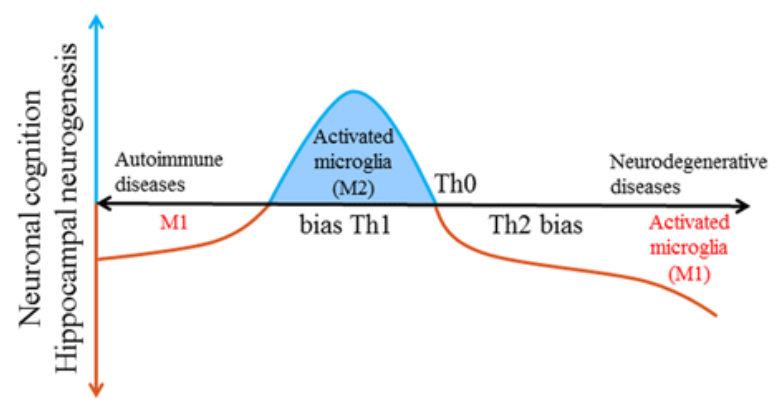

Figure 1: A simplified diagram describing relations between hippocampal microglia phenotype, systemic Th1/Th2 bias and neurobehavioral functions under in terms of physiological conditions and pathological conditions. (a) Blue/red colors represent increased/decline in hippocampal neurogenesis, respectively. (b) Systemic Th1/Th2 cytokine balance correlates with hippocampal neurogenesis [8-10], followed by M2 (alternatively activated) or M1 (classically activated) polarization of microglia in the present study. (c) There is less likely exist a linear relationship between them on basis of continuous invasion of Th1 cells in autoimmune diseases involved with cognitive decline, although systemic Th1 bias is positively associated with increased in neural proliferation [35]. (d) Proper Th2 bias or strong Th2 inflammation is associated with cognitive decline [36].

\section{Conclusion}

This review collectively illustrates the role for systemic Th1/Th2 balance in the regulation of adult hippocampal neurogenesis. It is attempting assume that changes of immune status in periphery regulate brain functions. Although Th1-type cytokines (IFN- $\gamma$, TNF-a et al.) are regard as pro-inflammatory factors implicated in the detrimental effects of neural proliferation and Th2-type cytokines (IL-4, IL-10 et al.) are anti-inflammatory factors, it is likely that proper cytokine balance is sufficient to modulate hippocampal neurogenesis for normal homeostasis in brain. In our recent studies, influenza vaccination including $A(\mathrm{H} 1 \mathrm{~N} 1)$ vaccine and Seasonal vaccine elicit Th1 like serum cytokine response accompanied by a remarkable neurobeneficial profile of cytokines and neurotrophic factors in the hippocampus $[10,12]$. Notably, systemic Th1/Th2 bias in this review is not pathological, but in normal range. Thus, altered adult hippocampal neurogenesis is transient both in pregnancy and exercise models. However, the fortissimo or excessive Th1/Th2 unbalance in autoimmune disease and neurodegenerative disease was, thus, not within the scope of the present investigation. Even so, the excessive
Th1/Th2 unbalance may still correlate to adult hippocampal neurogenesis (Figure 1).

\section{References}

1. Abbott NJ, Rönnbäck L, Hansson E (2006) Astrocyte-endothelial interactions at the blood-brain barrier. Nat Rev Neurosci 7: 41-53.

2. Engelhardt B, Ransohoff RM (2005) The ins and outs of T-lymphocyte trafficking to the CNS: anatomical sites and molecular mechanisms. Trends Immunol 26: 485-495.

3. Kleine TO, Benes L (2006) Immune surveillance of the human central nervous system (CNS): different migration pathways of immune cells through the blood-brain barrier and blood-cerebrospinal fluid barrier in healthy persons. Cytometry. Part A: the journal of the International Society for Analytical Cytology 69:147-151.

4. Schwartz M, Kipnis J (2011) A conceptual revolution in the relationships between the brain and immunity. Brain Behav Immun 25: 817-819.

5. Wolf SA, Steiner B, Wengner A, Lipp M, Kammertoens T, et al. (2009) Adaptive peripheral immune response increases proliferation of neural precursor cells in the adult hippocampus. FASEB J 23: 3121-3128.

6. Monje ML, Toda H, Palmer TD (2003) Inflammatory blockade restores adult hippocampal neurogenesis. Science 302: 1760-1765.

7. Musaelyan K, Egeland M, Fernandes C, Pariante CM, Zunszain PA, et al. (2014) Modulation of adult hippocampal neurogenesis by early-life environmental challenges triggering immune activation. Neural Plast 2014: 194396.

8. Palumbo ML, Trinchero MF, Zorrilla-Zubilete MA, Schinder AF, Genaro AM (2012) Glatiramer acetate reverts stress-induced alterations on adult neurogenesis and behavior. Involvement of Th1/Th2 balance. Brain Behav Immun. 26: 429-438.

9. Palumbo ML, Canzobre MC, Pascuan CG, Ríos H, Wald M, et al. (2010) Stress induced cognitive deficit is differentially modulated in $\mathrm{BALB} / \mathrm{c}$ and C57Bl/6 mice: correlation with Th1/Th2 balance after stress exposure. J Neuroimmunol 218: 12-20.

10. Xia Y, Qi F, Zou J, Yao Z (2014) Influenza A (H1N1) vaccination during early pregnancy transiently promotes hippocampal neurogenesis and working memory. Involvement of Th1/Th2 balance. Brain Res 1592: 34-43.

11. Baum M, Muller-Steinhardt M, Liesen H, Kirchner H (1997) Moderate and exhaustive endurance exercise influences the interferon-gamma levels in whole-blood culture supernatants. Eur J Appl Physiol Occup Physiol 76:165-169.

12. Xia Y, Qi F, Zou J, Yang J, Yao Z (2014) Influenza vaccination during early pregnancy contributes to neurogenesis and behavioral function in offspring. Brain Behav Immun 42: 212-221.

13. He F, Zou J-T, Zhou Q-F, Niu D-L, Jia W-H (2014) Glatiramer acetate reverses cognitive deficits from cranial-irradiated rat by inducing hippocampal neurogenesis. J Neuroimmunol 271: 1-7.

14. Rolls A, Schori H, London A, Schwartz M (2008) Decrease in hippocampal neurogenesis during pregnancy: a link to immunity. Mol Psychiatry 13: 468-469.

15. Piao HL, Tao Y, Zhu R, Wang SC, Tang CL, et al. (2012) The CXCL12/ CXCR4 axis is involved in the maintenance of Th2 bias at the maternal/ fetal interface in early human pregnancy. Cell Mol Immunol 9: 423-430.

16. Teixeira L, Moreira J, Melo J, Bezerra F, Marques RM, et al. (2015) Immune response in the adipose tissue of lean mice infected with the protozoan parasite Neospora caninum. Immunology .

17. Guha SK, et al. (2014) Single episode of mild murine malaria induces neuroinflammation, alters microglial profile, impairs adult neurogenesis, and causes deficits in social and anxiety-like behavior. Brain Behav Immun 42: 123-137.

18. Deng Y, Jing Y, Campbell AE, Gravenstein S (2004) Age-related impaired type $1 \mathrm{~T}$ cell responses to influenza: reduced activation ex vivo, decreased expansion in CTL culture in vitro, and blunted response to influenza 
Citation: Qi F, Xia Y, Zuo Z, Wu Y, Yang J, et al. (2015) Immune-Based Modulation of Adult Hippocampal Neurogenesis, Link to Systemic Th1/Th2 Balance. J Vaccines Vaccin 6: 274. doi:10.4172/2157-7560.1000274

Page 4 of 4

vaccination in vivo in the elderly. The Journal of Immunology 172 3437-3446.

19. Bloemen K, Verstraelen S, Van Den Heuvel R, Witters H, Nelissen I, et al. (2007) The allergic cascade: review of the most important molecules in the asthmatic lung. Immunol Lett 113: 6-18.

20. Villeda SA, Luo J, Mosher KI, Zou B, Britschgi M, et al. (2011) The ageing systemic milieu negatively regulates neurogenesis and cognitive function. Nature 477: 90-94.

21. Cakman I, Rohwer J, Schütz RM, Kirchner H, Rink L (1996) Dysregulation between TH1 and TH2 T cell subpopulations in the elderly. Mech Ageing Dev 87: 197-209.

22. Rink L, Cakman I, Kirchner H (1998) Altered cytokine production in the elderly. Mech Ageing Dev 102: 199-209.

23. Uciechowski P, Kahmann L, Plümäkers B, Malavolta M, Mocchegiani E, et al. (2008) TH1 and TH2 cell polarization increases with aging and is modulated by zinc supplementation. Exp Gerontol 43: 493-498.

24. Kahmann L, Uciechowski P, Warmuth S, Malavolta M, Mocchegiani E, et al. (2006) Effect of improved zinc status on T helper cell activation and TH1/TH2 ratio in healthy elderly individuals. Biogerontology 7: 429-435.

25. Park JH, Park Ok, Cho JH, Chen BH, Kim IH, et al. (2014) Antiinflammatory effect of tanshinone I in neuroprotection against cerebra ischemia-reperfusion injury in the gerbil hippocampus. Neurochem Res 39: 1300-1312.

26. Jiang $\mathrm{Y}$, Zou $\mathrm{Y}$, Chen S, Zhu C, Wu A, et al. (2013) The antiinflammatory effect of donepezil on experimental autoimmune encephalomyelitis in C57 BL/6 mice. Neuropharmacology 73: 415-424.

27. Neurath MF, Finotto S, Glimcher LH (2002) The role of Th1/Th2 polarization in mucosal immunity. Nat Med 8: 567-573.

28. Mosmann TR, Sad S (1996) The expanding universe of T-cell subsets: Th1, Th2 and more. Immunol Today 17: 138-146.
29. Lisak RP, Nedelkoska L, Studzinski D, Bealmear B, Xu W, et al. (2011) Cytokines regulate neuronal gene expression: differential effects of Th1, Th2 and monocyte/macrophage cytokines. J Neuroimmunol 238: 19-33.

30. Martino M, Rocchi G, Escelsior A, Fornaro M (2012) Immunomodulation Mechanism of Antidepressants: Interactions between Serotonin/Norepinephrine Balance and Th1/Th2 Balance. Curr Neuropharmacol 10: 97-123.

31. Beck RD, Wasserfall C, Ha GK, Cushman JD, Huang Z, et al. (2005) Changes in hippocampal IL-15, related cytokines, and neurogenesis in IL-2 deficient mice. Brain Res 1041: 223-230.

32. Wong G, Goldshmit Y, Turnley AM (2004) Interferon-gamma but not TNF alpha promotes neuronal differentiation and neurite outgrowth of murine adult neural stem cells. Exp Neurol 187: 171-177.

33. Baron R, Nemirovsky A, Harpaz I, Cohen H, Owens T, et al. (2008) IFNgamma enhances neurogenesis in wild-type mice and in a mouse model of Alzheimer's disease. FASEB J 22: 2843-2852.

34. Nunan R, Sivasathiaseelan H, Khan D, Zaben M, Gray W (2014) Microglial VPAC1R mediates a novel mechanism of neuroimmunemodulation of hippocampal precursor cells via IL-4 release. Glia 62: 1313-1327.

35. Schwartz M, Baruch K (2014) The resolution of neuroinflammation in neurodegeneration: leukocyte recruitment via the choroid plexus. EMBO J 33: 7-22.

36. Baruch K, Ron-Harel N, Gal H, Deczkowska A, Shifrut E, et al. (2013) CNS-specific immunity at the choroid plexus shifts toward destructive Th2 inflammation in brain aging. Proc Natl Acad Sci U S A 110: 2264-2269. 